\title{
Vibration Damping in Ni-Mn-Ga/PU Polymer Composites
}

\author{
Racil Jeya Geetha ${ }^{1,2}$, R. Senthur Pandi ${ }^{1}$, S. Seenithurai ${ }^{1}$, S. Vinodh Kumar ${ }^{1}$, M. Muthuraman ${ }^{1}$, \\ J. Thampi Thanka Kumaran', M. Mahendran",* \\ ${ }^{1}$ Smart Materials Lab, Department of Physics, Thiagarajar College of Engineering, Madurai, 625015, India \\ ${ }^{2}$ Department of Physics, Nesamony Memorial Christian College, Marthandam, 629165, India
}

\begin{abstract}
Ni-Mn-Ga ferromagnetic shape memory alloy is mainly focused from the class of active materials because of their large field induced strain. They exhibit strains up to $10 \%$ with respect to the applied magnetic field through twin boundary motion in the martensite phase. This is the main advantage of this material which could overcome the frequency bound of the conventional shape memory alloys. Ni-Mn-Ga FSMAs show much large field-induced strain at low frequencies than do other active materials such as piezoelectrics or magentostrictors. When the twin boundaries are moving back and forth, the hysteresis connected to this motion provides vibration damping part of the damping bases on the magnetoelastic properties of the materials. The excellent damping properties of Ni-Mn-Ga alloys are bonded with the correct polymer matrix makes possible to develop new damping materials which are effective, less expensive and easier to form than bulk Ni-Mn-Ga. They can suit well to be used in the correct site for effective damping in special engineering applications. In this article, we have prepared the composite materials and reported its basic characterization and the damping behavior of composite materials.
\end{abstract}

Keywords Vibration Damping, Ni-Mn-Ga, FSMA Polymer Composites

\section{Introduction}

Ferromagnetic Shape Memory Alloys (FSMAs) are well known for their giant Magnetic-Field Induced Strain (MFIS), which makes them potential candidates for applications such as actuators and sensor[1,2]. Almost 10\% of MFIS has been reported in martensitic Ni-Mn-Ga in 2002[1]. The FSMA differs from the conventional SMA by its twin boundary motion in the martensitic phase rather than the structural change[1-4]. The operating frequency of many conventional shape memory alloys is limited to some $\mathrm{Hz}$ whereas FSMA can be used in $\mathrm{KHz}$. The strain recovery of these materials is due to the twin boundary movement, which is very different from the domain growth mechanism of magnetostrictive material. The maximum strain of this material is determined by its crystal structure[5].

The High positioning accuracy of Ni-Mn-Ga makes them as a promising candidate for potential applications[6]. Actuation and sensing nature of this material are limited by their brittleness. The advantages like high frequency performance and the large MFIS of Ni-Mn-Ga will be limited. In actuation applications, it needs a

* Corresponding author:

manickam-mahendran@tce.edu (M. Mahendran)

Published online at http://journal.sapub.org/cmaterials

Copyright (C) 2011 Scientific \& Academic Publishing. All Rights Reserved compressive loading to recover its original shape. The crystal form of this alloy cannot be used for cyclic actuators and dampers. So the brittleness should be overcome without affecting the strain output of this material. These disadvantages are partially overcome by embedding the $\mathrm{Ni}-\mathrm{Mn}-\mathrm{Ga}$ particle into the polymer matrix. There are several reports about the polymer matrix Ni-Mn-Ga composites by various research groups[7-15]. The polymer magnetic composite have many advantages such as light weight, low cost and design flexibilities.

The polymer composites with aligned particles are expected as a better solution to resolve the brittleness behavior. The composite is significantly more flexible than the crystalline materials which allow cyclic compression and expansions. Ni-Mn-Ga reinforced polymer composites and their mechanical behavior are reported. The particles in the composite are respond to the mechanical stress with twin boundary motion. The proper alignment with magnetic curing leads to the particle interaction and twin boundary motion. Cyclic actuation, vibration damping, magnetization in polymer composites, twin boundary movement in polymer composites and stress- strain studies for energy absorption applications were already carried out[8-18]. In the present work, owing to the want of much needed scientific data on the Ni-Mn-Ga/PU composites, a new material having good mechanical damping has been designed and developed. The composite of this kind has been chosen, as it is useful in potential applications like 
vibration damping applications[7-18].

\section{Experimental Procedure}

\subsection{Polycrystal Preparation}

The polycrystalline ingots are produced using the arc-melting furnace. The arc melting chamber is fleshed off with the pure argon for three to four time to remove the as much as air inside. While the melting process the chamber is completely filled with argon. It helps the arc to travel and reach the sample in the chamber. The raw materials of the systems are taken with high purity in the stoichiometric ratio. After the first melt, all the ingots are reverted in their same place and melted once again in the argon atmosphere. This melting procedure is repeated upto six times with replacing the ingots in all the dimensions. This multiple time melting procedure is done to achieve the homogeneity in the sample. Once the melt is completed the samples are taken out of the chamber and sealed in a quartz tube with argon atmosphere for thermal annealing. Thermal treatment for the phase transformation is done in the samples. The samples are kept in a box furnace with $1100^{\circ} \mathrm{C}$ for 10 days. After that quenching in the systems are done to stabilize the phase.

\subsection{Composite Preparation}

For the composites preparation samples with similar sizes cut down from the crystals and crushed into powders. The crystals are changed in to particles using the ball milling process (Retsch PM100). The mortar grinding and spark eroded powder based composite are already reported. The milling process produces rigid solid particles unlike the hollow one in the spark erosion. The particle sizes are dependent on the milling time. The milled particles of the $\mathrm{Ni}-\mathrm{Mn}-\mathrm{Ga}$ are mixed with polymer resin completely. The magnetic particle and polymer mixture must be optimized. The polymer composites should be made in only martensite temperatures. So that, the twin boundaries in the particles are present and also the particles with easy axis alignment are possible due to their crystal structures.

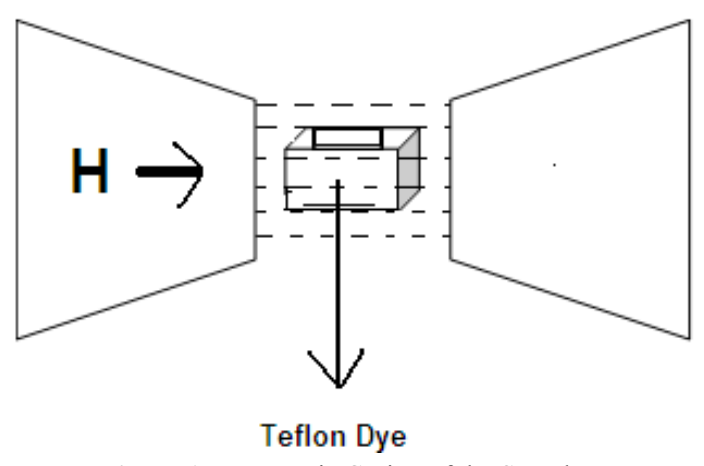

Figure 1. Magnetic Curing of the Samples

The composite preparation is similar to the Hand lay-Up method. This particle mixed slurry is taken into a mold and this mold is placed in the magnetic field of $4 \mathrm{KOe}$ with a
Laboratory Electro Magnet. The sample is completely cured in the magnetic field as shown in figure 1. The magnetic curing process aligns the magnetic particles in the slurry to a particular direction. The magnetic curing is done for half an hour. The composites of $20 \%$ volume of Ni-Mn-Ga powder and 24\% volume of Ni-Mn-Ga powder are prepared. Then the samples are cut down to different dimensions for different characterization.

The alignment of the particle depends on the magnetic field and magneto crystalline anisotropy of the sample. The high magneto crystalline anisotropy along the c- axis will help to align the particles in the matrix. The short c- axis in the tetragonal martensite will act as the easy axis of magnetization. The easy axis orientation of the particles can be made by two ways. The first one is magnetic field curing during the composite preparation. In this process, the curing axis will act as the easy axis of magnetization. The second one is applying mechanical stress in the desired direction so as to form the easy axis magnetization. Now, the particle alignment at preferred orientation will make a way for the twin boundaries movement.

\subsection{Damping Setup}

In the setup, the power supply is coupled with a function generator which acts as the input for the piezostack actuator. The actuator is in direct contact with sample, the input voltage with a particular frequency for which the actuator converts the signal into corresponding mechanical vibrations. These mechanical vibrations pass through the sample and transmitted signal is converted by the sensor to electrical signal. PZT ceramic sheet of $0.5 \mathrm{~mm}$ thickness is used as sensor. The longitudinal stress waves passed through the samples with different input peak to peak voltages (Vpp) are received by the PZT sensor. The voltage produced by sensor is picked up through a lock-in amplifier.

\section{Results and Discussion}

\subsection{Structural Studies}

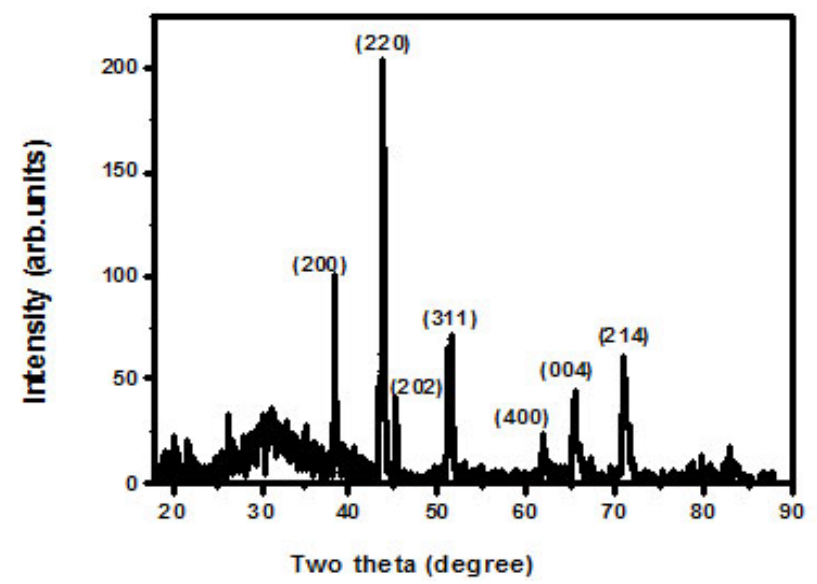

Figure 2. Powder X-ray Diffraction Pattern of the Ni-Mn-Ga Polycrystal 
X-ray diffraction pattern is used to study the structure of the prepared sample. The sample is crushed into powders. Then the crushed powder is annealed in an inert atmosphere at $400^{\circ} \mathrm{C}$ for 10 hours to remove the residual stresses. The powder x-ray diffraction data is collected at room temperature using an $18 \mathrm{KW}$ copper rotating anode-based Powder diffractometer fitted with a graphite monochromator in the diffracted beam. The data collection in the $2 \theta$ range of $10-90^{\circ}$ is carried out using scintillation counter with the scan rate of $2 \% \mathrm{~min}$.

The dominant (220) peak reflects the L21 structure formation in these materials. At lower angles the (200) reflection peak in the samples. The (200) peak confirm the order of the structure. At higher angles (311) (400) (004) and (214) peaks are the signature of the martensite phase of the alloy. So, the presence of the martensite phase in the room temperature is also confirmed by the pattern. The (110) martensite peak is not observed in the samples. Some time the higher noise in our sample will diminish that peak. So the martensite phase formation and the L21 structure are identified in these studies. The (220) reflection peak doesn't show any peak splitting. Usually the peak splitting in this peak reflects the orthorhombic structure. We did not observe such kind of splitting in our peaks. So the tetragonal martensite structure is confirmed by its lattice parameters.

\subsection{Micro-Structural Studies}

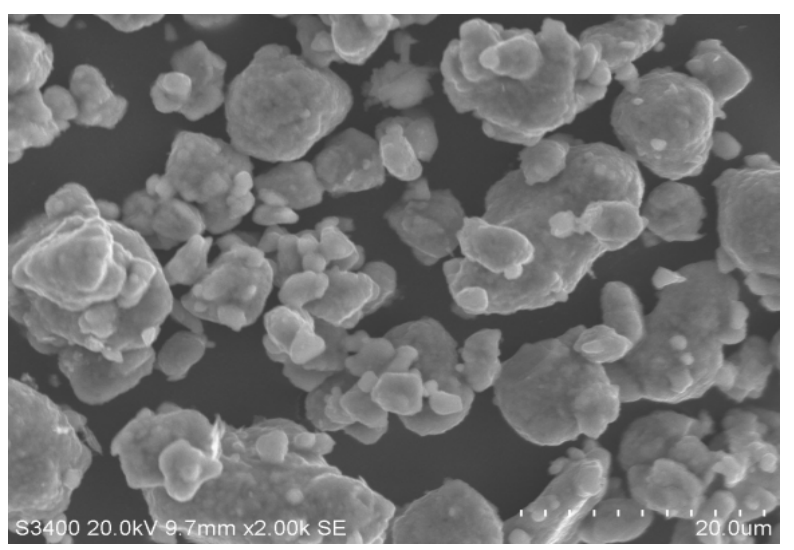

Figure 3. SEM image of Ni-Mn-Ga Crystalline Powder

Scanning Electron Microscope (SEM) images show the surface morphology of the particles and their existence in the composite sample. The figure 3 shows the SEM micrograph of the milled Ni-Mn-Ga Particle. It can be observed that the particles are random in their size and shape. These shape difference is due to the random collision between the particles and the balls in the milling process. The average particle size is less than 15 micro meter. The stress produced in those particles is relieved by the thermal treatment. These particles are mixed with the polyurethane slurry.

The SEM image of Ni-Mn-Ga/PU composite is shown in figure 4 . The Ni-Mn-Ga particles are not spread uniformly in the matrix of the sample. The particles are shown in the figure itself. Non uniform alignment is due to the processing difficulties. The particle distribution must be controlled when they are mixed with the matrix slurry. Very low viscous of the polymer will give troubles while the composite preparation. The proper viscous of the slurry will help to form better uniformity of the particles. The magnetic particle alignment in a uniform way will lead to the better magnetic properties.

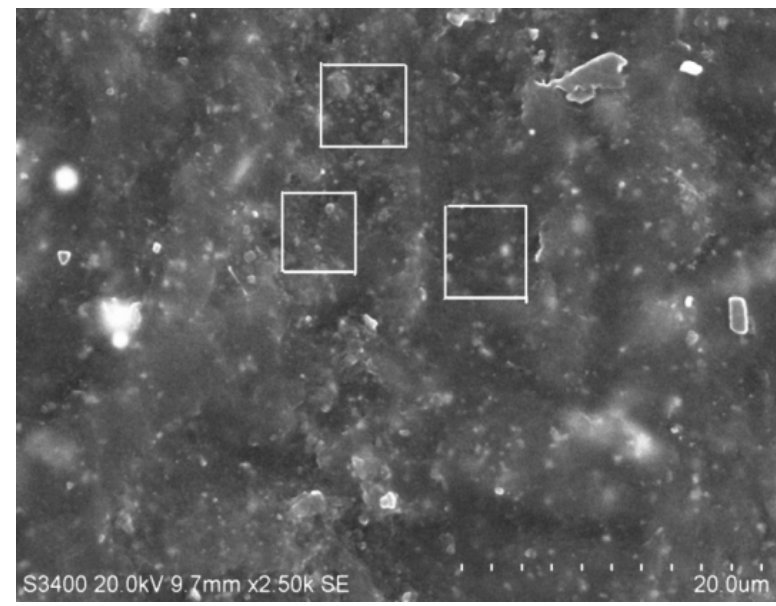

Figure 4. SEM image of Ni-Mn-Ga/Polymer Composite

The magnetic response of these composites is also dependent on the particles size. The Nano Scaled particle size is undesirable one for this work. In the case of nano scale, the ferromagnetic material is going to exhibit the superpara magnetism. This will affect the particle orientation during curing process and the magnetic response of the composite is reduced. So the particles in the micro scale are desirable for these composites.

\subsection{Elemental Analysis using Scanning Electron Microscope}

Figure 5 a) and b) show the SEM images of the $\mathrm{Ni}-\mathrm{Mn}-\mathrm{Ga} / \mathrm{PU}$ composites. The SEM pictures can be easily identified by the separate compound analysis images. The $\mathrm{Ni}$ content and the Carbon content of the polymer composite is identified in separate pictures. The elemental analysis is an evident one for the particle and matrix identification in the SEM pictures. The grey matrix ball like structures is the Ni-Mn-Ga particles present in the matrix. The glittered and most sprayed material is identified as polymer matrix. So, the alignment of the particles is not clearly viewed in the above picture. It confirms the particulate and matrix material.

\subsection{Magnetic Studies}

The magnetic measurement is done in the Lakhshore 7404 Vibrating Sample Magnetometer (VSM). The magnetic hysteresis loops of the sample are shown in the figure 6 . The hysteresis curve indicates the ferromagnetic nature of the polymer composites. The saturation magnetization is one of the important factors for the magnetic field induced strains. The saturation magnetization will reflect the fer- 
romagnetic order. It can be evaluated by the magnetization curve. Figure 6 shows graph between the field versus magnetization of the composite samples. This hysteresis loop show magnetic behavior of the particles in the matrix material. The saturation magnetization is very low when comparing with the single crystals of this alloy. It is due to variation from the bulk materials to particle ones and their immersion in polymer matrix of the composites[19]. The particle ratio in the polymer is one of the important parameter for the magnetic behavior of these composites.

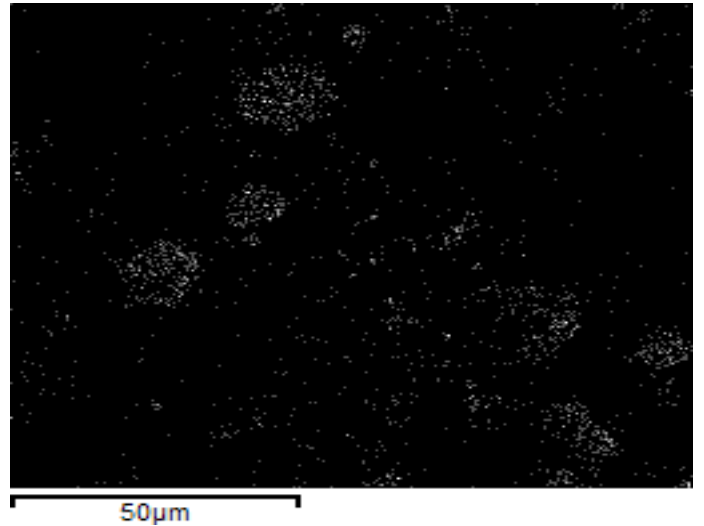

Figure 5 a). Elemental Analysis studies using Scanning Electron Microscope (Ni content)

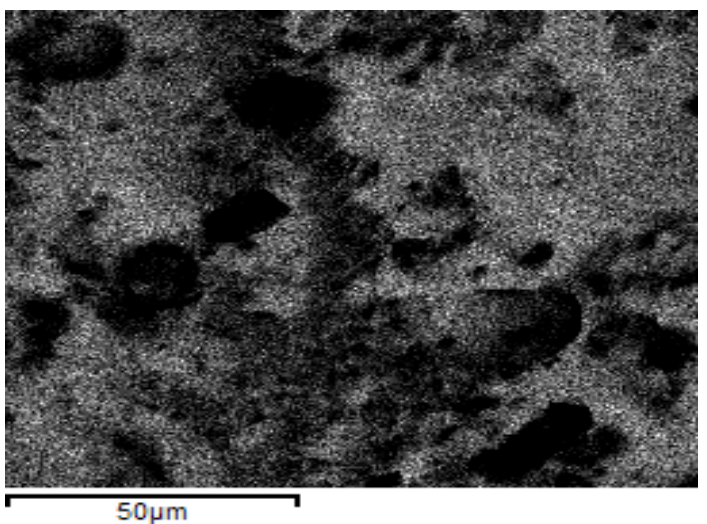

Figure 5 b). Elemental Analysis studies using Scanning Electron Microscope (Carbon content (Polymer))

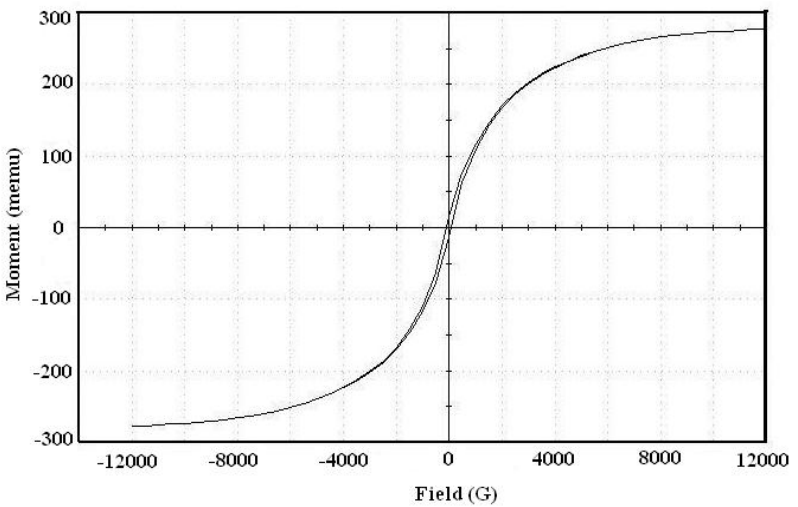

Figure 6. Magnetic Studies of Ni-Mn-Ga particulate based polymer composites

The magnetic properties like coercivity, retentivity and saturation magnetization are derived from the hysteresis curve. The coercivity, retentivity and the saturation magnetization of the composite sample is $95.433 \mathrm{G}, 14.811$ memu and 277.56 memu respectively.

\subsection{Damping Studies}

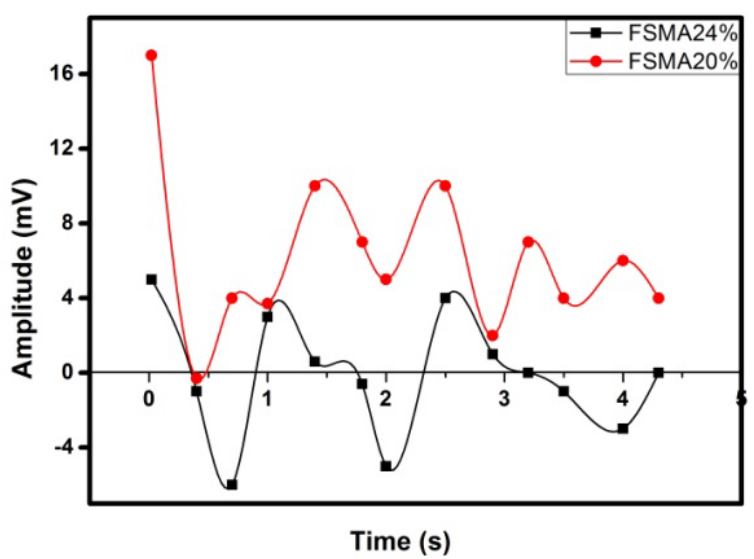

Figure 7. The graph is plotted between the voltage and time parameters. The graph shows damping ability between the two closely optimized composites

Figure 7 shows two different samples of FSMA composites of $20 \%$ and $24 \%$ ratio fillers. The graph is drawn between the time period and voltage at the frequency of 1 $\mathrm{KHz}$. The percentage of the filler particles in the composite makes different in the absorption of the stress waves. The transmission time of the acoustic wave depends on the impedance of the materials[20,21]. The stress amplitudes slowly decrease when the time extends. The amplitude of the wave slowly decreased due parameters like internal friction. The FSMA composite of $24 \%$ filler shows low transmitted wave comparing to the others. It shows a high absorbing quality. The performance of the composite will be enhanced by optimizing the parameters like filler percentage and particle size.

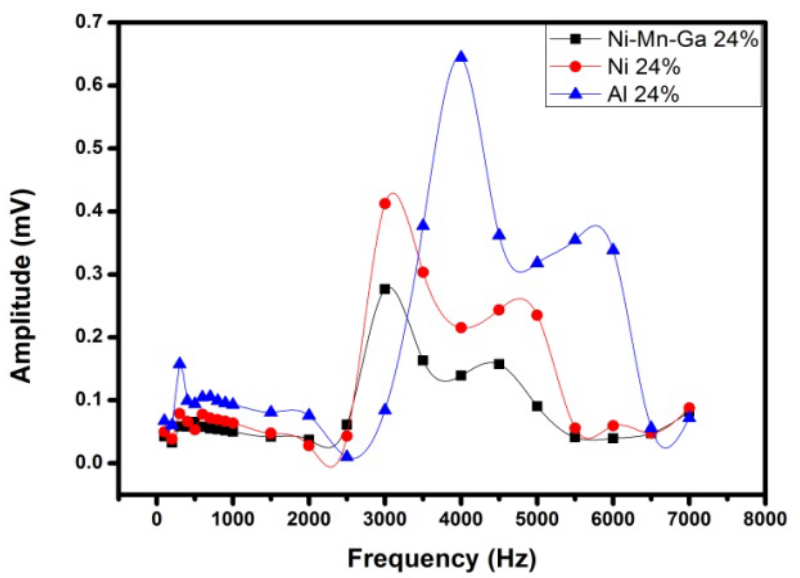

Figure 8. Damping Studies of Different metal particulate based polymer composites

The figure 8 differentiates well about the different particulate composites. The materials with different magnetic behavior and microstructure are chosen for comparison. The Ni-Mn-Ga/PU composite shows higher damping ca- 
pability than the others. The aluminium is chosen for non-magnetic material and the $\mathrm{Ni}$ is chosen for its magnetic property. The aluminium cannot be aligned in the magnetic field. In the case of $\mathrm{Ni}$, it can be aligned in the magnetic field but it doesn't have twin boundaries like as the Ni-Mn-Ga. In the above graph shows the damping ability of these composites cured under the magnetic field. The Ni-Mn-Ga shows high damping capacity than the other two materials. It reveals the importance of the alignment of the particles and the presence of twin boundaries. Though the modulus and density of the materials determine the impedance of the composites, the alignment and microstructure of the particulate also has to be focused. It can be expected that increasing twin boundaries present in the material will enhance the damping mechanism of the composites. The increase in the no. of twin boundaries can be made of the compositional change of the alloy. The polymer matrix of the composites can be chosen by their stiffness as per their applications. Thus, the damping behavior of the entire composite can be tailored as per industrial requirements.

\section{Conclusions}

The polycrystalline Ni-Mn-Ga alloy is prepared and the room temperature martensite is confirmed. Then, the Ni-Mn-Ga/PU Polymer composite is fabricated to overcome the brittle nature of this active material. The magnetic particles are tried to align in the polymer matrix during curing process. The alignment of the particles is studies directly in the SEM results. The alignment may be formed either by optimizing the magnetic curing parameters or mechanical stress at particular direction. $24 \%$ volume fraction of the alloy in the polymer matrix is optimized through the damping studies. It is concluded that this polymer composite can be tailored to a very good absorbing material for the specific vibration damping applications.

\section{ACKNOWLEDGMENTS}

MM thanks Dr. Robert C. O’Handley for his scholarly guidance and introducing him to the subject. The authors thank MIT Magnetic Materials group for their suggestions in composite preparation techniques and damping set up.

\section{REFERENCES}

[1] Ullakko, K., Huang, J. K., Kantner, C., and O'Handley R. C., 1996, Giant magnetic- field-induced strain in NiMnGa seven-layered martensitic phase, Applied Physics Letters, 69, 1966-1968

[2] Chernenko, V. A., Besseghini, S., Müllner, P., Kostorz G.,
2007, Ferromagnetic Shape Memory Materials: Underlying Physics and Practical Importance, Sensor Letters, 5, 229-233

[3] Mullner, P., Chernenko, V. A., Wollgarten, M., and Kostorz, G., 2002, Large Cyclic deformation of a Ni-Mn-Ga shape memory alloy induced by magnetic fields, Journal of Applied Physics, 92 ,6708-6713

[4] Mullner, P., and Ullakko, K., 1998, The force of a magnetic/electric field on a twinning dislocation, Physica Status Solidi (b), 208, R1-R2

[5] Kokorin, V. V., Chernenko, V. A., Cesari, E., Pons, J., and Segui, C., 1996, Pre-martensitic state in Ni-Mn-Ga alloys, Journal of Physics: Condensed Matter, 8, 6457-6463

[6] Yuanchang Liang., Yauso Luga., and Minoru Taya., 2006, Design of membrane Actuator Based on Ferromagnetic Shape Memory alloy composite for synthetic jet applications, Sensors and Actuators A, 125, 512-518

[7] Hideki Hosoda., Shinsuke Takeuchi., Tomonari Inamura., Kenji Wakashima., 2004, Material design and shape memory properties of smart composites composed of polymer and ferromagnetic shape memory alloy particles, Science and Technology of Advanced Materials, 5, 503-509

[8] Aaltio, I., Lahelin, M., Soderberg, O., Heczko, O., Lofgren, B., Ge, Y., Seppala, J., Hannula, S. P., 2009, DMA testing of Ni-Mn-Ga/polymer composites, Composites: Part A, 40, $125-129$

[9] Lahelin, M., Aaltio, I., Heczko, O., Soderberg, O., Ge, Y., Lofgren, B., Seppala, J., Hannula, S. P., 2008, Temperature dependence of the damping properties of Ni-Mn-Ga alloys, Materials Science and Engineering A, 481-482, 314-317

[10] Eric Gans., and Greg P Carman., 2006, Cyclic actuation of Ni-Mn-Ga composites, Journal of Applied Physics, 99, 084905-1-4

[11] Jorge Feuchtwanger., Kelli Griffin., Jiankang Huang., David Bono., Robert C. O' Handley., Samuel M. Allen., 2004, Mechanical energy absorption in Ni-Mn-Ga polymer composites, Journal of Magnetism and Magnetic Materials, 272-276, 2038-2039

[12] Jorge Feuchtwanger., Marc L Richard., Yun J Tang, Ami E. Berkowitz., Robert C.O' Handley., Samuel M. Allen.,2005, Large energy absorption in $\mathrm{Ni}-\mathrm{Mn}-\mathrm{Ga} /$ polymer composites, Journal of Applied Physics, 97, 10M319-1-10M319-3

[13] Jorge Feuchtwanger., Sadie Michael., Jiankang Huang., David Bono., Robert C. O' Handley., Samuel M. Allen., and Catherine Jenkins., 2003, Energy absorption in Ni-Mn-Gapolymer composites, Journal of Applied Physics, 93, $8528-8530$

[14] Scheerbaum, N., Hinz, D., Gutfleisch, O., Muller, K.H., and Schultz, L., 2007, Textured polymer bonded composites with Ni-Mn-Ga magnetic shape memory particles, Acta Materialia, 55, 2707-2713

[15] Scheerbaum, N., Heczko, O., Liu, J., Hinz, D., Schultz, L., and Gutfleisch, O., 2008, Magnetic field-induced twin boundary motion in polycrystalline Ni-Mn-Ga fibres, New Journal of Physics, 10, 073002-1-8

[16] Xiaogang Sun., Chaoying Xie., 2007, Damping Characteristics of A NiMnGa/Polymer Composite Material, Mate- 
rials Science Forum, 561-565, 697-699

[17] Manickam Mahendran., Jorge Feuchtwanger., and Robert C.O. Handley., 2011, Acoustic energy absorption in $\mathrm{Ni}-\mathrm{Mn}-\mathrm{Ga}$ /polymer composites, Journal of Magnetism and Magnetic Materials, 323, 1098-1100

[18] Senthur Pandi, R., Chokkalingam, R., and Mahendran, M., 2011, Temperature Dependent damping properties on $\mathrm{Ni}-\mathrm{Mn}-\mathrm{Ga} / \mathrm{PU}$ Polymer Composites, Bulletin of Materials Science, 34, No. 4, 739-743

[19] Ramalingam Chokkalingam., Rajasabai Senthur Pandi., and Manickam Mahendran., 2011, Magnetomechanical behaviour of $\mathrm{Fe} / \mathrm{PU}$ magnetorheological elastomers, Journal of Composite Materials, 45, 1545-1552

[20] Senthur Pandi, R., Chokkalingam, R., Vallal Peruman, K., Kodi Pandyan, R., and Mahendran., M., 2010, Amplitude Stress in Ni-Mn-Ga Polymer Composites Bonded with PZT Sensors and Actuators, Integrated Ferroelectrics, 121, $77-85$

[21] Senthur Pandi, R., Chokkalingam, R., and Mahendran, M., 2011, Stress Wave Propagation in Ni-Mn-Ga Ferromagnetic Shape Memory Alloys, Japanese Journal of Applied Physics, 50, 075801-1-4 